\title{
Hepatitis B vaccine immunogenicity among nurses of a hospital
}

\author{
V Piratheepkumar ${ }^{1}$, S Kulendran $^{1}$, S Nadarajah $^{2}$, K Murugananthan $^{1}$ \\ (Index words: hepatitis B, hepatitis B surface antibody (HBsAb), hepatitis B vaccination, nursing staff)
}

\begin{abstract}
The aim of this study was to determine the factors affecting hepatitis $B$ vaccine immunogenicity among nursing staff of Teaching Hospital, Jaffna. 152 nursing staffs were recruited from April to July 2013. Those who received three doses had $7.7 \%$ failure rate in developing protective immune response. Failure rates were $20 \%$ in those who received one dose and $10.6 \%$ in two doses. Following vaccination, participants who had chickenpox had a failure rate of $75 \%(n=13 ; p=0.02)$. Pregnancy significantly impaired protective immunity among those who received two doses $(p=0.03)$. Three doses of hepatitis $B$ vaccine may not be sufficient to protect the vaccinees. Therefore assessment of HBsAb titres is warranted after vaccination.
\end{abstract}

Ceylon Medical Journal 2014; 59: 59-60

\section{Introduction}

Hepatitis B infection is a significant health problem worldwide. Hepatitis B virus (HBV) causes up to $80 \%$ of all cases of hepatocellular carcinoma worldwide. About two billion people worldwide have been infected with the virus, and about 350 million live with chronic infection [1]. Every year there are over four million new acute clinical cases of hepatitis $\mathrm{B}$, and one million people die from chronic active hepatitis, cirrhosis and primary liver cancer every year [2]. In Sri Lanka, epidemiological surveys of the community and in blood donors demonstrated that the prevalence of chronic HBV infection was low, ranging between $0.7 \%$ and $3 \%$ [3].

Health care professionals are at high risk of exposure to $\mathrm{HBV}$ and therefore $\mathrm{HBV}$ vaccine is mandatory for all health care workers worldwide. However little is known about the protection provided by HBV vaccine in Sri Lanka. This study was designed to address this gap. The aim of this study was to determine hepatitis B surface antibody (HBsAb) titres and factors influencing the efficacy of the vaccine among nursing staff at the Teaching Hospital, Jaffna (THJ), Sri Lanka vaccinated for hepatitis B.

\section{Methods}

Blood donor data from 28,511 donors at the Jaffna Regional Blood Centre, THJ were analysed to determine the prevalence of HBV infection in the population. The study population included 152 hepatitis $\mathrm{B}$ vaccinated nursing staff from THJ, who were selected by simple random sampling. Blood samples were tested for hepatitis B surface antibody (HBsAb) titre using a qualitative ELISA (Monolisa Anti-HBs Plus; sensitivity 99.5\%, specificity $99.4 \%$ ) in the Microbiology laboratory, University of Jaffna. Protective antibody titre levels equal or more than $10 \mathrm{mIU} / \mathrm{mL}$ for HBsAb were considered to be protective [2]. Host related factors such as age, sex, co-morbidities, medications, previous accidental exposure to hepatitis B infection and vaccination related factors (number of doses taken, receiving booster doses, receiving institute) were obtained by a self-administered questionnaire. The study was carried out between April and July 2013. Chi-square test $\left(\chi^{2}\right.$ test) was used to identify associations. The study was approved by the Ethics Committee, University of Jaffna.

\section{Results}

Of the 152 nursing staff, $55.9 \%$ were aged between 20-29 years, $57.9 \%$ were women. All nursing staff had been vaccinated with Shanvac-B recombinant plasma derived vaccine in the deltoid muscle. All participants received their vaccination in government hospitals in Sri Lanka, and 143 (94.1\%) had received their vaccination at the THJ.Among the participants $10(106.6 \%)$ received only one dose, 47 (30.9\%) received two doses, and 95 $(62.5 \%)$ received all three doses.

Among the participants who received 3 doses 7 $(7.73 \%)$ failed to develop protective immunity. Failure rates among those who received one dose was $20 \%(\mathrm{n}=2)$ and two doses $10.6 \%(n=5)$. After two doses of vaccination, there was significant deterioration in protective immunity after four years $(p<0.05)$. In individuals who received 3 doses the protective immunity did not reduce significantly after four years (Figure 2). Of the 28,511 blood donors 32 $(0.11 \%)$ were positive for HBV. Co-morbidities (diabetes mellitus, hypertension, bronchial asthma) were not

Departments of ${ }^{1}$ Pathology, ${ }^{2}$ Community and Family Medicine, Faculty of Medicine, University of Jaffna, Sri Lanka.

Correspondence: KM, e-mail: <kalamathy6@gmail.com>. Received 6 November 2013 and revised version accepted 20 March 2013. Competing interests: none declared. 


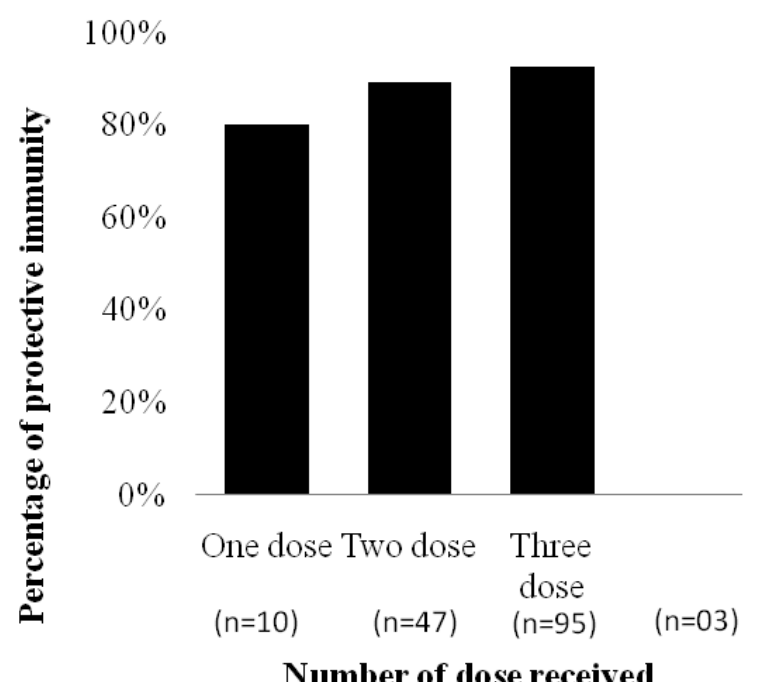

Figure 1. The percentage of individuals in each category who develop protective immunity.

significantly associated with protective immunity to HBV following immunisation. Participants who were treated for chickenpox with acyclovir $(n=13)$ following immunisation had a $75 \%$ failure rate in developing protective immunity $(p=0.02)$. Pregnancy significantly impaired the protective immunity $(p=0.03)$ among individuals. Who had only two doses.

\section{Discussion}

Health care professionals should have adequate protective immunity to HBV. Since two doses of vaccine did not completely protect pregnant women against HBV, a full vaccination schedule should be recommended for this group. All individuals who return a negative antibody test following completion of vaccination should be recommended to have a booster dose of HBV vaccine. Rates of impaired immunity among pregnant females are similar to those found in a British study [4]. It was found that those who received two doses had statistically significant impairment in their post vaccination immunity. In the current study, participants who received three doses had $100 \%$ protection following vaccination.

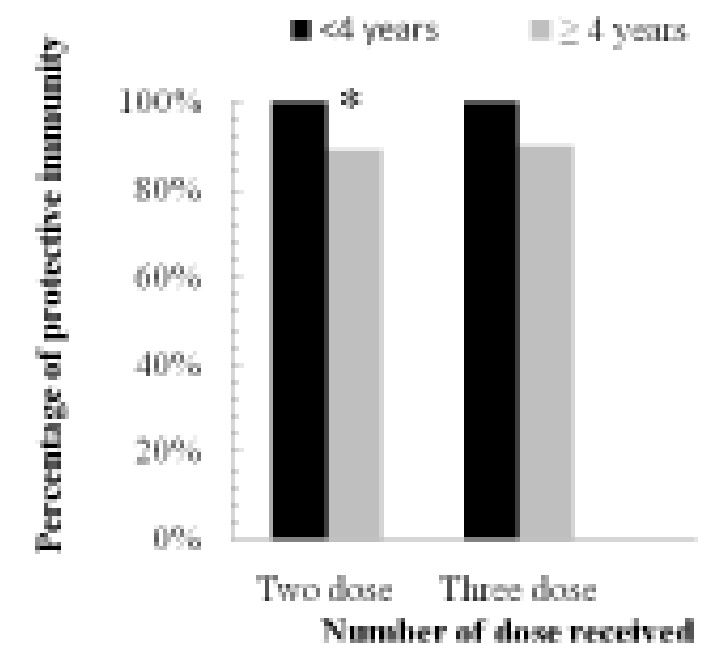

Figure 2. Change in protective immunity with time.

According to our knowledge, failure following acyclovir treatment has not been previously reported. A more comprehensive study in a larger population is warranted to address the mechanisms that may be involved in this process.

\section{Acknowledgements}

We thank Professor Natkunam Ketheesan from the Australian Institute of Tropical Health and Medicine for helping to write this manuscript.

\section{References}

1. Immunization Hand Book. Epidemiology Unit, Ministry of Health, Sri Lanka, 2012; 38: 31.

2. Hepatitis B (2002) World Health Organization WHO/CDS/ CSR/LYO/2002.

3. Perera J, Perera B, Gamage S. Seroconversion after hepatitis $B$ vaccination in healthy young adults, and the effect of a booster dose. Ceylon Medical Journal 2002; 47: 6-8.

4. Ingardia CJ, Kelley L, Steinfeld JD, Wax JR. Hepatitis B vaccination in pregnancy: factors influencing efficacy. Obstetrics and Gynecology 1999; 93: 983-6. 